\title{
Expression of LAT1 and 4F2hc in Gastroenteropancreatic Neuroendocrine Neoplasms
}

\author{
YOSUKE HORITA $^{1}$, KYOICHI KAIRA ${ }^{2 *}$, TOMONORI KAWASAKI ${ }^{3 *}$, YOSHIAKI MIHARA ${ }^{1}$, \\ SHINICHI SAKURAMOTO ${ }^{4}$, SHIGEKI YAMAGUCHI ${ }^{4}$, KOJUN OKAMOTO ${ }^{4}$, SHOMEI RYOZAWA $^{5}$, \\ YOSHIKATSU KANAI ${ }^{6}$, MASANORI YASUDA ${ }^{3}$ and TETSUYA HAMAGUCHI ${ }^{1}$
}

\begin{abstract}
Departments of ${ }^{1}$ Gastroenterological Oncology, ${ }^{2}$ Respiratory Medicine, ${ }^{3}$ Pathology, ${ }^{4}$ Gastroenterological Surgery, and ${ }^{5}$ Gastroenterology, International Medical Center, Saitama Medical University, Saitama, Japan;

${ }^{6}$ Department of Bio-system Pharmacology, Graduate School of Medicine, Osaka University, Osaka, Japan
\end{abstract}

\begin{abstract}
Background/Aim: Little is known about the expression of L-type amino acid transporter 1 (LAT1) and $4 F 2 h c$ in gastroenteropancreatic-neuroendocrine neoplasms (GEP-NENs). Hence, we conducted a study to verify the clinicopathological significance of LAT1 and $4 F 2 h c$. Patients and Methods: Tissues from 126 patients with GEP-NENs were collected between August 2007 and August 2019 at our institution. We evaluated LAT1 and $4 F 2$ hc expression by immunohistochemistry, and examined their clinical significance. Results: No statistically significant associations were observed between LAT1 expression and the different NENs. Expression of $4 F 2 h c$ was significantly different between neuroendocrine tumour (NET)-G1, NET-G2, and NET-G3 ( $p=0.029)$, and was significantly associated with vascular invasion $(p=0.044)$ and the Ki-67 index $(p=0.042)$. Conclusion: No association between LAT1 expression and malignant features in GEP-NENs was observed. However, an association between $4 F 2 h c$ expression and the potential of malignancy in GEP-NENs was evident.
\end{abstract}

This article is freely accessible online.

*These Authors contributed equally to this study.

Correspondence to: Yosuke Horita, MD, Department of Gastroenterological Oncology, Comprehensive Cancer Center, International Medical Center, Saitama Medical University, 1397-1 Yamane, Hidaka-City, Saitama 350-1298, Japan. Tel: +81 429844111, Fax: +81429840432, e-mail: york01@ saitamamed.ac.jp; Kyoichi Kaira, MD, Ph.D., Department of Respiratory Medicine, Comprehensive Cancer Center, International Medical Center, Saitama Medical University, 1397-1 Yamane, Hidaka-City, Saitama 350-1298, Japan. Tel: +81 429844111, Fax: +81 429844741, e-mail: kkaira1970@yahoo.co.jp

Key Words: LAT1, 4F2hc, CD98, neuroendocrine tumour, gastroenterology, amino acid transporter.
Cancer cells require nutrients, such as glucose, amino acids, and fat, to survive and grow within human tissues. Glucose metabolism plays a crucial role in tumour progression and angiogenesis. However, amino acid metabolism is also necessary for tumour survival; cancer cells uptake many amino acids via amino acid transporters (1-3). In particular, L-type amino acid transporter 1 (LAT1) is associated with the uptake of essential neutral amino acids into tumour cells, and is extensively expressed in most human cancers (2-14). According to evidence from many clinicopathological studies, high expression of LAT1 is closely correlated with tumour proliferation, angiogenesis, metastasis, and survival (2-14). The 4F2 heavy chain (4F2hc: CD98), known as the heavy chain of LAT1, functions cooperatively in the uptake of amino acids (3). Therefore, cooperative expression of 4F2hc with LAT1 has been identified as a significant prognostic marker in several human cancers $(6,9,13,14)$. Previous investigations have confirmed different percentages of LAT1 expression based on histological types $(4,11,12)$. However, little is known about the clinicopathological significance of LAT1 and 4F2hc expression in patients with neuroendocrine neoplasms (NENs) arising from gastroenteropancreatic (GEP) organs.

NEN is a rare condition arising from a variety of organs, including pancreas, gastrointestinal tract, and lungs. GEPNENs are classified into two categories: neuroendocrine tumours (NETs) and neuroendocrine cancers (NECs). The World Health Organization (WHO) has categorized NENs into four groups, namely low (grade 1), intermediate (grade 2 ), and high (grade 3 ) grade NETs, and poorly differentiated NECs $(15,16)$, based on Ki-67 labelling index rate and mitotic figure. Histological classification of lung NENs consists of typical carcinoid, atypical carcinoid, and large and small NECs (17). Furthermore, NETs are categorised as functional or non-functional, depending on their hormonal activity. Tumour cell proliferation, determined by the Ki-67 labelling index as assessed by immunohistochemistry, has been correlated with tumour grade $(15,16)$. However, there 
are no established biomarkers to predict the efficacy and treatment outcomes, or to elucidate the pathogenesis of NENs. As described above, it has been reported that amino acid transport systems play a crucial role in cellular proliferation. Further, LAT1 is significantly correlated with Ki-67, tumour differentiation, and survival. We previously reported that LAT1 expression tended to increase from lowgrade to high-grade lung NETs, and that LAT1 expression in large cell NECs (LCNECs) was closely linked to lymph node metastasis and poor outcomes (12). Moreover, we have also reported that $4 \mathrm{~F} 2 \mathrm{hc}$ expression tends to increase from lowgrade to high-grade NETs (18). However, it remains unclear whether the expression of LAT1 and 4F2hc is related to the clinicopathological features in patients with GEP-NENs.

Hence, we conducted a clinicopathological study to evaluate the expression of LAT1 and 4F2hc in patients with GEP-NENs.

\section{Patients and Methods}

Patients. In this retrospective study, paraffin-embedded tissue specimens from patients with NENs were collected between August 2007 and August 2019 at our institution. Medical records of 166 patients with histologically confirmed NENs were analysed retrospectively. Of them, 25 patients were excluded because of inadequate tumour specimens, whereas 15 others were excluded because of other types of advanced cancer at the time of diagnosis, resulting in a final total of 126 patients being included in the study.

A complete work-up, including pathological assessment, clinical course, and physical examination, was performed for all cases, and was reviewed by pathologists and expert physicians (YH, TK, MY, and $\mathrm{TH}$ ). All patients were classified according to the WHO criteria. According to histological assessment, NENs were classified as NETG1, NET-G2, NET-G3, or NEC, and tumour cell proliferation was determined by counting the Ki-67 labelling index. Clinicopathological variables, including age, gender, histological type, tumour size, tumour grade, lymph node metastasis, lymphatic invasion, vascular invasion, pathological disease staging, and outcome, were obtained from pathological reports and medical records. This study was approved by the institutional ethics committee of the Saitama Medical University International Medical Center (Approval number: 19-228; Approval date: 2019/11/13). All procedures followed were in accordance with the Helsinki Declaration of 1964 and its later versions.

Immunohistochemistry. LAT1 expression was determined immunohistochemically by incubating tumour samples with rabbit monoclonal antibody against LAT1 (J-Pharma, Tokyo, Japan), at a dilution of 1:5,000 in phosphate-buffered saline containing $0.1 \%$ bovine serum albumin, at $4^{\circ} \mathrm{C}$ overnight, followed by incubation at room temperature for $30 \mathrm{~min}$. An affinity-purified goat polyclonal antibody raised against a peptide located at the carboxy terminus of 4F2hc (1:200 dilution; Santa Cruz Biotechnology, Inc., Tokyo, Japan) of human origin was used for studying 4F2hc expression. The reaction was visualized using a Histofine Simple Stain MAXPO (Multi) Kit (Nichirei, Tokyo, Japan), following the manufacturer's instructions, as described previously (19). Negative controls were incubated without the primary antibody, and no detectable staining was evident. Expression of LAT1 and 4F2hc was
Table I. Patient characteristics $(n=126)$.

\begin{tabular}{llc}
\hline Category & & $\mathrm{N}$ \\
\hline Age, median year (range) & & $62.0(27-80)$ \\
Gender & Male/Female & $81 / 45$ \\
Stage* & $1 / 2 / 3 / 4$ & $90 / 15 / 14 / 7$ \\
Histology & NET G1/G2/G3 & $98 / 16 / 4$ \\
& NEC & 8 \\
Primary site & Rectum & 73 \\
& Gastro-esophageal & 22 \\
& Small bowel & 20 \\
& Pancreas & 9 \\
& Colon & 1 \\
& Liver & 1 \\
Method of sample collection & ESD or EMR** & 80 \\
& Surgery & 36 \\
& Biopsy & 10 \\
\hline
\end{tabular}

*TNM Classification of Malignant Tumours, $8^{\text {th }}$ edition; **ESD: Endoscopic submucosal dissection; EMR: endoscopic mucosal resection; NET: neuroendocrine tumour; NEC: neuroendocrine cancer.

considered positive only if distinct membrane staining was observed. The percentage of LAT1 and 4F2hc staining was scored as follows: $1,0-10 \% ; 2,11-25 \% ; 3,26-50 \%$; and $4,51-100 \%$. Staining intensity was not considered while assessing staining outcomes. Expression was considered positive when tumours contained cancer cells that were assigned staining scores of 3 or 4 .

Immunohistochemical staining for $\mathrm{Ki}-67$ was performed following the procedure described previously (19). A murine monoclonal antibody against Ki-67 (Dako, Glostrup, Denmark; 1:40 dilution) was used. Areas of the immuno-stained sections with high cell density were assessed. All epithelial cells with nuclear staining of any intensity were defined as having high expression. Approximately 1000 nuclei were counted on each slide. Proliferative activity was assessed as the percentage of Ki-67stained nuclei (Ki-67 labeling index) in the sample. The sections were evaluated using a light microscope in a blinded fashion by at least two of the authors. In case of a discrepancy, both investigators simultaneously evaluated the slides until a consensus was reached. Both investigators were blinded to patient outcomes.

Statistical analysis. Statistical analyses were performed using Pearson's chi-square test for categorical variables. Correlations were analysed using the Mann-Whitney $U$-test and Spearman's rank test. The Kaplan-Meier method was used to estimate survival as a function of time, and differences in survival were analysed using the log-rank test. Overall survival (OS) was defined as the time from treatment initiation to death from any cause. Statistical significance was set at $p<0.05$. The hazard ratio was estimated using a Cox proportional hazards model. All statistical analyses were performed using IBM SPSS ver. 25.0 software (SPSS Inc., Chicago, IL, USA).

\section{Results}

Patient characteristics. The characteristics of the 126 enrolled patients are shown in Table I. The cohort consisted of 81 men and 45 women, with a median age of 


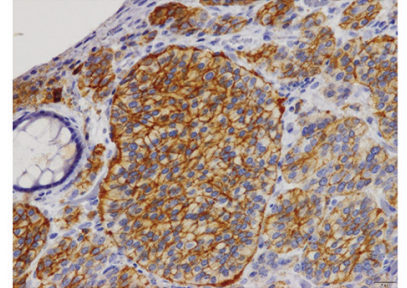

(A) Score 4

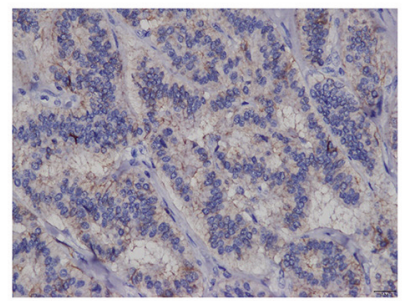

(C) Score 2

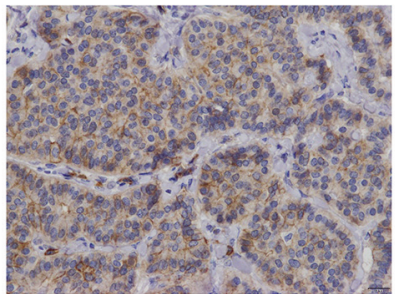

(B) Score 3

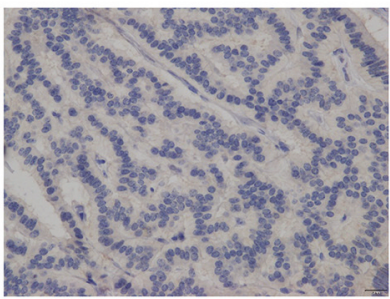

(D) Score 1

Figure 1. Immunostaining scores for LAT1 in rectal neuroendocrine tumour (NET)-G1 cases for grade $4(A)$, grade $3(B)$, grade $2(C)$, and grade $1(D)$. Staining score was defined as follows: 1, 0-10\%; 2, 11-25\%; 3, 26-50\%; 4, 51-100\% stained. A score of 3 or 4 was considered as positive.

62.0 years $($ range $=27-80$ years $)$. Histological classification indicated NET-G1 in 98 specimens, NET-G2 in 16, NETG3 in 4, and NEC in 8 . The most common primary site was the rectum, followed by the oesophagus, stomach, small bowel, and pancreas.

Patient demographics and immunohistochemical findings. Representative images for different immunohistochemical staining scores of LAT1 and 4F2hc expression in paraffinembedded tissue specimens from tumours at the primary sites are presented in Figures 1 and 2. Demographic distribution of the different variables according to LAT1 and 4F2hc expression is listed in Table II. The degree of positive expression of LAT1 in NET-G1, NET-G2, NET-G3, and NEC was $50.0 \%$ (49/98), $43.8 \%$ (7/16), $75.0 \%$ (3/4), and $50.0 \%(4 / 8)$, respectively. No statistically significant associations were observed between LAT1 expression and the different variables (Table II).

The degree of positive $4 \mathrm{~F} 2 \mathrm{hc}$ expression was $65.3 \%$ $(64 / 98)$ in NET-G1, $87.5 \%(14 / 16)$ in NET-G2, $100 \%(4 / 4)$ in NET-G3, and $62.5 \%(5 / 8)$ in NEC. Positive $4 \mathrm{~F} 2 \mathrm{hc}$ expression was significantly higher in patients with vascular invasion (Table II). Only NET cases were analysed (Table III), and NEC cases were excluded. Positive expression of LAT1 was closely associated only with 4F2hc expression ( $p=0.046$, Pearson's chi-square test). In contrast, positive $4 \mathrm{~F} 2 \mathrm{hc}$ expression was significantly associated with vascular invasion and

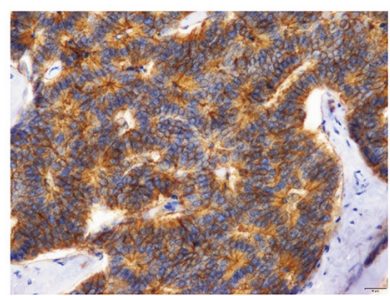

(A) Score 4

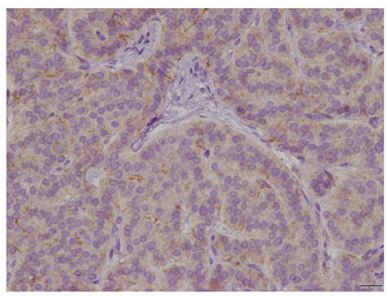

(C) Score 2

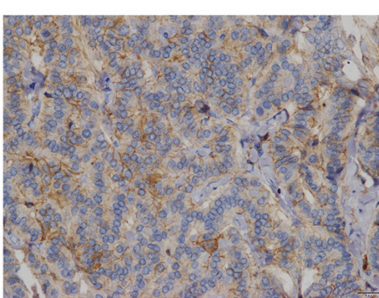

(B) Score 3

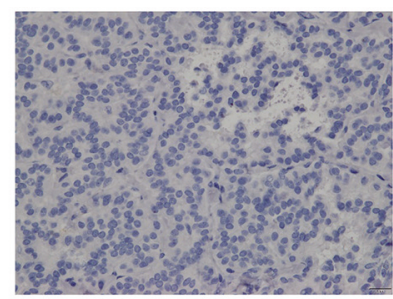

(D) Score 1
Figure 2. Immunostaining scores for $4 F 2$ hc in rectal neuroendocrine tumour (NET)-G1 cases for grade $4(A)$, grade $3(B)$, grade $2(C)$, and grade $1(D)$. Staining score was defined as follows: 1, 0-10\%; 2, 11-25\%; 3, 26-50\%; 4 , $51-100 \%$ stained. A score of 3 or 4 was considered as positive.

histological NET grading (excluding NEC). There was a statistically significant difference in $4 \mathrm{~F} 2 \mathrm{hc}$ expression between NET-G1 and NET-G2/G3 ( $p=0.029$, Pearson's chisquare test).

Further, no correlation was found between the Ki-67 index and LAT1 expression, whereas a statistically significant correlation between $4 \mathrm{~F} 2 \mathrm{hc}$ expression and the Ki-67 index was observed ( $p=0.042$, Mann-Whitney $U$-test) (Figure 3).

Clinical course. Among the 126 patients, 114 underwent curative resection, and 6 had recurrence. All recurrences were observed in NET-G2 and NEC patients (three patients each). Of the 14 patients who received chemotherapy, six were recurrent cases, whereas seven were stage 4 , and one was inoperable stage $2 \mathrm{G} 1$ due to complications. Of the 14 patients who received chemotherapy, seven received platinum-based chemotherapy, five received everolimus, and a somatostatin analogue was administered to six (there were overlaps in patient treatments). Of the eight patients who died, the cause was primary disease in six cases, and other diseases in two cases.

Survival analysis. There was a significant difference in OS between patients with NET-G1 and others (NET-G2/3 and NEC) $(p=0.001$, log-rank test) (Figure 4$)$. Neither LAT1 nor 4F2hc expression was significantly associated with OS, but the hazard ratio for $4 \mathrm{~F} 2 \mathrm{hc}$ expression was 3.15 , which indicates a possibility of association (Figure 5). 
Table II. Expression of LAT1 and $4 F 2 h c$.

\begin{tabular}{|c|c|c|c|c|c|c|c|}
\hline \multirow[t]{2}{*}{ Variables } & \multirow{2}{*}{$\begin{array}{c}\text { Total } \\
\mathrm{n}=126\end{array}$} & \multicolumn{3}{|c|}{ LAT1 } & \multicolumn{3}{|c|}{ 4F2hc (CD98) } \\
\hline & & $\begin{array}{c}\text { Positive } \\
n=63\end{array}$ & $\begin{array}{c}\text { Negative } \\
n=63\end{array}$ & $p$-Value & $\begin{array}{c}\text { Positive } \\
n=87\end{array}$ & $\begin{array}{c}\text { Negative } \\
n=39\end{array}$ & $p$-Value \\
\hline Age $(<69 / \geq 69)$ years & $90 / 36$ & $46 / 17$ & $44 / 19$ & 0.693 & $63 / 24$ & $27 / 12$ & 0.715 \\
\hline Gender (male/female) & $81 / 45$ & $39 / 24$ & $42 / 21$ & 0.577 & $57 / 30$ & $24 / 15$ & 0.667 \\
\hline $\mathrm{T}$ factor $(\mathrm{T} 1-2 / \mathrm{T} 3-4)$ & $117 / 9$ & $57 / 6$ & $60 / 3$ & 0.299 & $81 / 6$ & $36 / 3$ & 0.873 \\
\hline $\mathrm{N}$ factor (present/absent) & $20 / 106$ & $12 / 51$ & $8 / 55$ & 0.330 & $16 / 71$ & $4 / 35$ & 0.248 \\
\hline Stage (I-II/III-IV) & $105 / 21$ & $51 / 12$ & $54 / 9$ & 0.473 & $70 / 17$ & $35 / 4$ & 0.196 \\
\hline Stage (I/II-IV) & $90 / 36$ & $44 / 19$ & $46 / 17$ & 0.693 & $59 / 28$ & $31 / 8$ & 0.180 \\
\hline Grading (G1/G2, G3 and NEC) & $98 / 28$ & $49 / 14$ & $49 / 14$ & 1.000 & $64 / 23$ & $34 / 5$ & 0.089 \\
\hline Primary tumor size $(<2.0 / \geq 2.0) \mathrm{cm}$ & $108 / 18$ & $53 / 10$ & $55 / 8$ & 0.611 & $73 / 14$ & $35 / 4$ & 0.387 \\
\hline $\begin{array}{l}\text { Primary site (Gastrointestinal NEN/ } \\
\text { Pancreatic NEN) }\end{array}$ & $116 / 9$ & $57 / 6$ & $59 / 3$ & 0.311 & $79 / 8$ & $37 / 1$ & 0.192 \\
\hline Lymphatic invasion (present/absent) & $30 / 96$ & $16 / 47$ & $14 / 49$ & 0.676 & $20 / 67$ & $10 / 29$ & 0.747 \\
\hline Vascular invasion (present/absent) & $34 / 92$ & $19 / 44$ & $15 / 48$ & 0.422 & $28 / 59$ & $6 / 33$ & $0.050 *$ \\
\hline LAT1 (positive/negative) & $63 / 63$ & & & & $48 / 39$ & $15 / 24$ & 0.083 \\
\hline 4F2hc (positive/negative) & $87 / 39$ & $48 / 15$ & $39 / 24$ & 0.083 & & & \\
\hline
\end{tabular}

LAT1: L-type amino acid transporter 1; 4F2hc: 4F2 heavy chain; NEN: neuroendocrine neoplasms. *Statistically significant as per Pearson's chisquare test; "One case of primary liver was excluded.

Table III. Expression of LAT1 and 4F2hc in NET (NEC excluded).

\begin{tabular}{|c|c|c|c|c|c|c|c|}
\hline \multirow[t]{2}{*}{ Variables } & \multirow{2}{*}{$\begin{array}{c}\text { Total } \\
\mathrm{n}=118\end{array}$} & \multicolumn{3}{|c|}{ LAT1 } & \multicolumn{3}{|c|}{ 4F2hc (CD98) } \\
\hline & & $\begin{array}{c}\text { Positive } \\
\mathrm{n}=59\end{array}$ & $\begin{array}{c}\text { Negative } \\
n=59\end{array}$ & $p$-Value & $\begin{array}{c}\text { Positive } \\
n=82\end{array}$ & $\begin{array}{c}\text { Negative } \\
n=36\end{array}$ & $p$-Value \\
\hline Age $(<69 / \geq 69)$ years & $88 / 30$ & $45 / 14$ & $43 / 16$ & 0.672 & $61 / 21$ & $27 / 9$ & 0.944 \\
\hline Gender (male/female) & $75 / 43$ & $36 / 23$ & $39 / 20$ & 0.566 & $53 / 29$ & $22 / 14$ & 0.714 \\
\hline $\mathrm{T}$ factor (T1-2/T3-4) & $114 / 4$ & $56 / 3$ & $58 / 1$ & 0.309 & $78 / 4$ & $36 / 0$ & 0.178 \\
\hline $\mathrm{N}$ factor (present/absent) & $12 / 106$ & $8 / 51$ & $4 / 55$ & 0.223 & $11 / 71$ & $1 / 35$ & 0.078 \\
\hline Stage (I-II/III-IV) & $105 / 13$ & $51 / 8$ & $54 / 5$ & 0.378 & $70 / 12$ & $35 / 1$ & 0.058 \\
\hline Stage (I/II-IV) & $90 / 28$ & $44 / 15$ & $46 / 13$ & 0.665 & $59 / 23$ & $31 / 5$ & 0.096 \\
\hline Grading (G1/G2 or G3) & $98 / 20$ & $49 / 10$ & $49 / 10$ & 1.000 & $64 / 18$ & $34 / 2$ & $0.029 *$ \\
\hline Primary tumor size $(<2.0 / \geq 2.0) \mathrm{cm}$ & $107 / 11$ & $52 / 7$ & $55 / 4$ & 0.342 & $72 / 10$ & $35 / 1$ & 0.105 \\
\hline $\begin{array}{l}\text { Primary site (Gastrointestinal NEN/ } \\
\text { Pancreatic NEN) }\end{array}$ & $110 / 8$ & $54 / 5$ & $56 / 3$ & 0.464 & $75 / 7$ & $35 / 1$ & 0.252 \\
\hline Lymphatic invasion (present/absent) & $25 / 93$ & $13 / 46$ & $12 / 47$ & 0.822 & $18 / 64$ & $7 / 29$ & 0.759 \\
\hline Vascular invasion (present/absent) & $27 / 91$ & $16 / 43$ & $11 / 48$ & 0.273 & $23 / 59$ & $4 / 32$ & $0.044 *$ \\
\hline LAT1 (positive/negative) & $59 / 59$ & & & & $46 / 36$ & $13 / 23$ & $0.046^{*}$ \\
\hline 4F2hc (positive/negative) & $82 / 36$ & $46 / 13$ & $36 / 23$ & $0.046^{*}$ & & & \\
\hline
\end{tabular}

LAT1: L-type amino acid transporter 1; 4F2hc: 4F2 heavy chain; NEN: neuroendocrine neoplasms. *Statistically significant using Pearson's chisquare test; \# One case of primary liver was excluded.

Recurrence. In the verification of postoperative recurrence, five of six $(83.3 \%)$ patients with recurrence displayed positive 4F2hc expression, whereas $4 \mathrm{~F} 2 \mathrm{hc}$ was negatively expressed in 72 of $108(66.7 \%)$ non-recurrence cases. However, there was no statistically significant correlation between tumour recurrence and 4F2hc expression ( $p=0.396$, Pearson's chi-square test).

\section{Discussion}

This is the first study to evaluate the expression of LAT1 and 4F2hc in GEP-NENs. LAT1 expression has been previously reported to be closely related to the malignancy and prognosis of various cancers (4-14). However, in our study, we did not find a tendency for malignant features to be associated with 

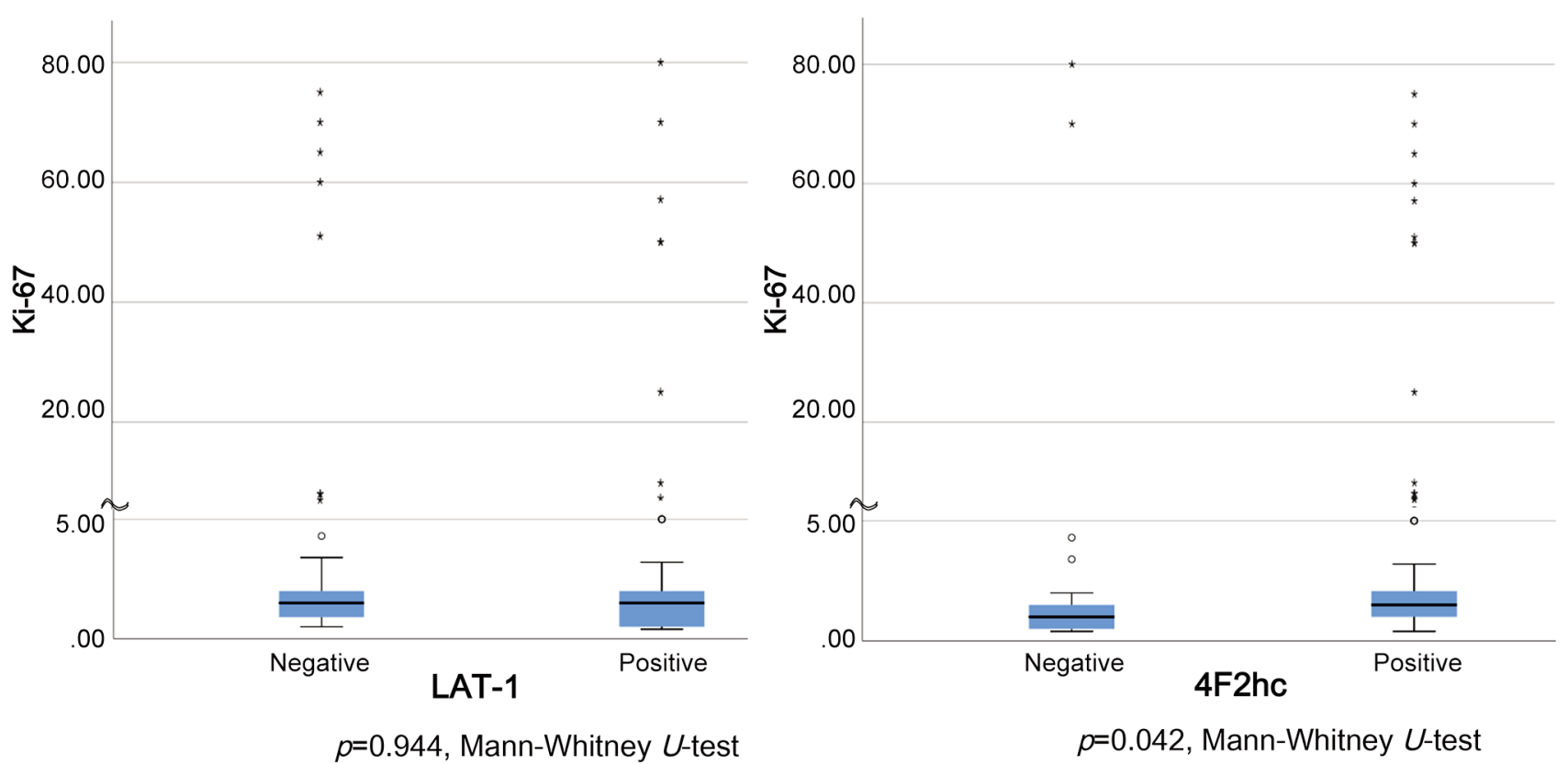

Figure 3. Correlation between Ki-67 index and LAT1 or 4F2hc expression. No correlation was found between Ki-67 index and LAT1 expression. However, a statistically significant correlation between $4 F 2 h$ expression and Ki-67 index was found ( $p=0.042$, Mann-Whitney U-test).

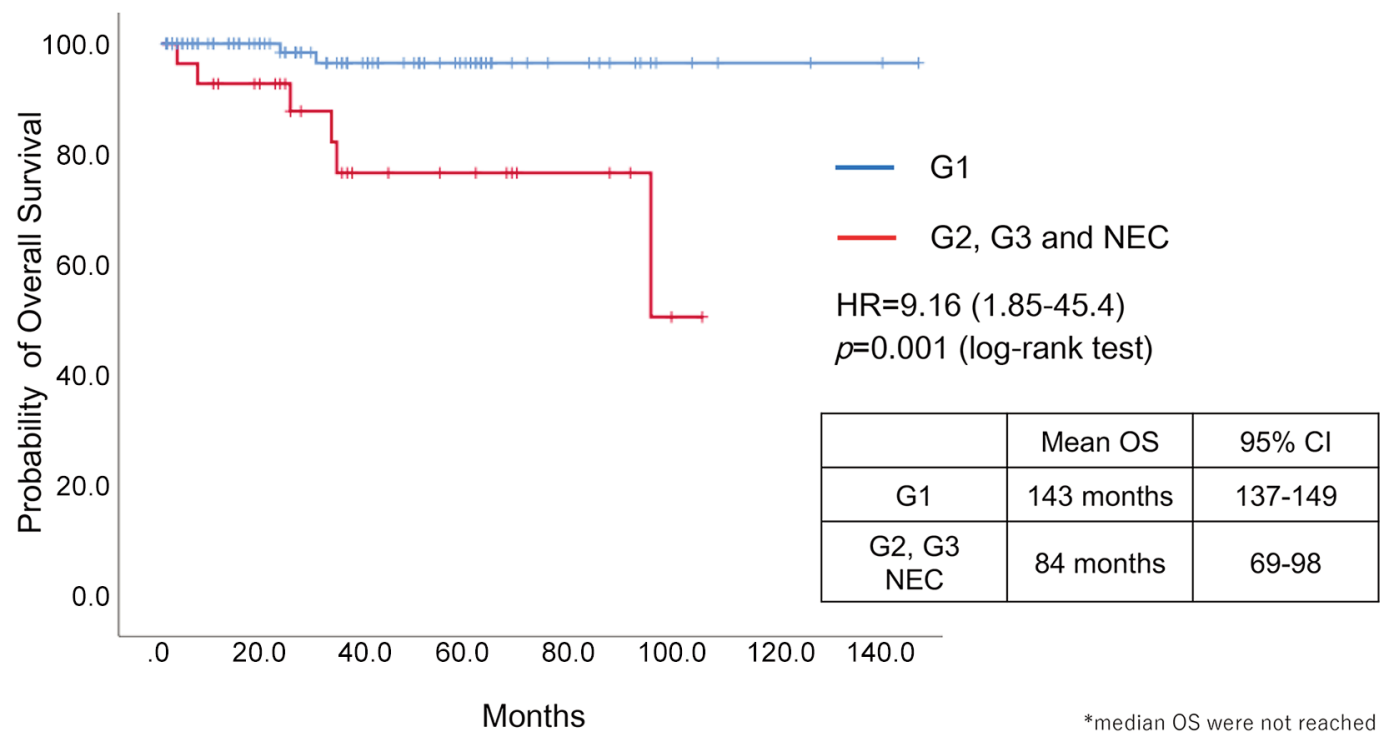

Figure 4. Overall survival according to histological grade. There was a significant difference in overall survival (OS) between patients with neuroendocrine tumour (NET)-G1 and others (NET-G2/3 and NEC) $(p=0.001, \log$-rank test).

the LAT1 expression level. In contrast, the expression of $4 \mathrm{~F} 2 \mathrm{hc}$ was associated with vascular invasion, NET grading, and tumour cell proliferation, as determined by the Ki-67 labeling index. 4F2hc is a heavy chain of LAT1, and functions cooperatively in the uptake of amino acids (3). Previous studies have shown that $4 \mathrm{~F} 2 \mathrm{hc}$ expression is closely correlated with tumour malignancy and prognosis $(6,7,9,13$, 18-20, 26). Furuya et al. reported that the prognosis tended to be worse when both LAT1 and 4F2hc were expressed (6). The expression rates of LAT1 and $4 \mathrm{~F} 2 \mathrm{hc}$ varied among different types of cancers, as follows: LAT1/4F2hc: breast cancer, $43 \% / 32 \%$ (6); pancreatic cancer, 53\%/57\% (9); non- 

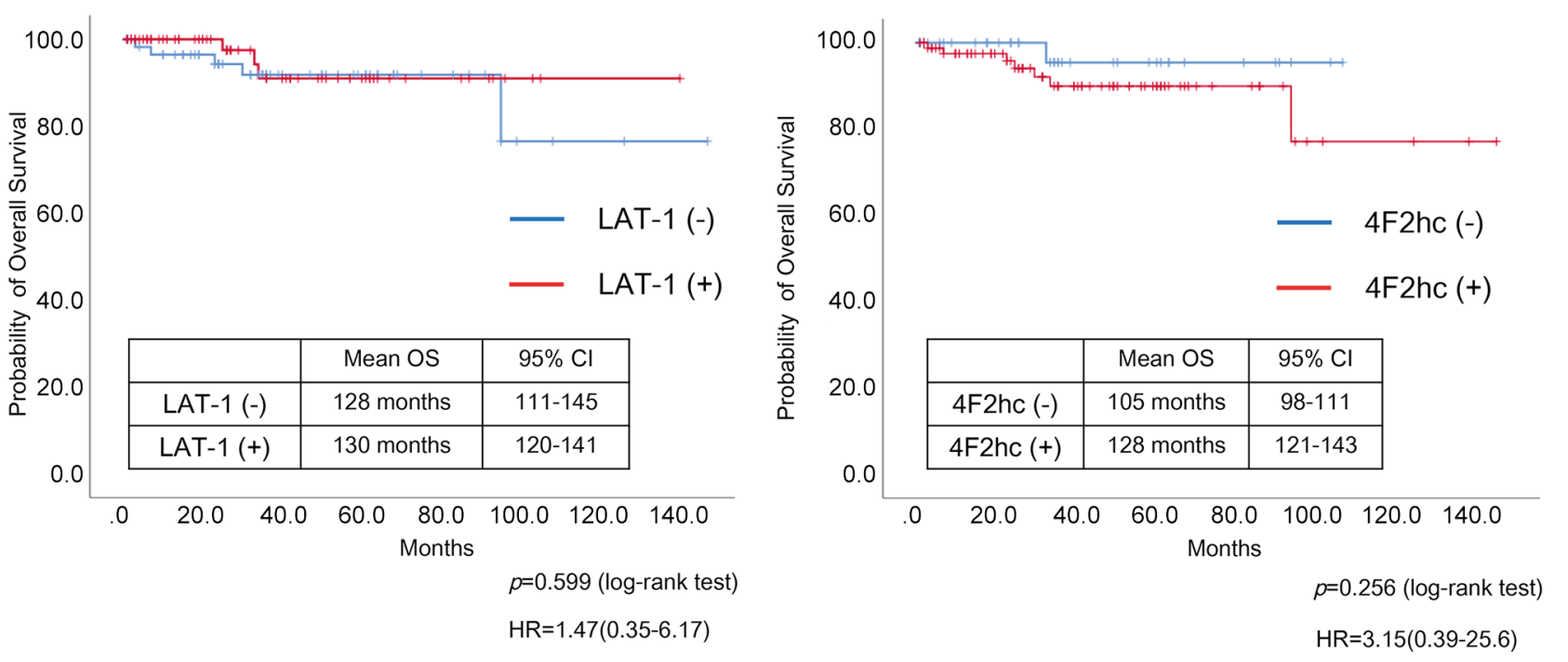

*median OS were not reached

*median OS were not reached

Figure 5. Overall survival according to LAT1 and $4 h 2 F c$ expression. LAT1 and $4 F 2 h c$ expression were not associated with OS ( $p=0.599$ and $p=0.256$, respectively, log-rank test).

small cell lung cancer, 37-60\%/34-47\% (10, 11, 19); colorectal cancer, $80 \% / 59 \%$ (13). In our study, the expression rate of $4 \mathrm{~F} 2 \mathrm{hc}$ was higher than that of LAT1 (LAT1/4F2hc: $50 \% / 69 \%$ ). GEP-NENs are heterogeneous neoplasms that are involved in the expression of related carcinogenic pathways (21). Although a relationship between LAT1 and 4F2hc expression was observed (Table III), the potential of malignancy for GEP-NENs was only associated with 4F2hc expression in this study. This discrepancy may be attributed to the stainability and heterogeneity of GEP-NENs.

The mammalian target of rapamycin (mTOR) signalling pathway receives inputs from amino acids, nutrients, growth factors, and environmental cues to regulate various fundamental cellular processes, including protein synthesis, growth, metabolism, aging, regeneration, and autophagy. Activation of the mTOR signalling is essential for growth promotion, and is implicated in human cancer, playing various roles in cell survival, cytoskeleton rearrangement, invasion, metastasis, anti-apoptosis, and inhibition of autophagy (22). LAT1, 4F2hc, and alanine-serine-cysteine amino acid transporter-2 (ASCT2) are closely related to the mTOR pathway $(5,11,13)$. Fuchs et al. hypothesized that mTOR regulates amino acid transporter gene expression and their transport to the plasma membrane in response to growth signals in cancer cells (5). Everolimus, an mTOR inhibitor, significantly prolonged progression-free survival among patients with progressive advanced pancreatic NETs in a phase 3 trial (RADIANT 3) (23). Furthermore, the efficacy of everolimus was demonstrated in the RADIANT 4 study, which was a phase 3 study in patients with gastrointestinal NEN (24). In both these pivotal clinical trials, the effect of everolimus was independent of histological malignancy. In our study, everolimus was administered to five patients, and the clinical response was confirmed in four patients, one of whom had partial response (PR), whereas three had stable disease. Positive expression of both LAT 1 and 4F2hc was observed in patients with PR.

In the present study, LAT1 and 4F2hc were positively expressed in the tumour cells of NENs. However, the positive expression of LAT1 was not linked to tumour progression and angiogenesis, in contrast to previous investigations. A close correlation between LAT1 and 4F2hc expression was observed in patients with NETs. However, a significant association between $4 \mathrm{~F} 2 \mathrm{hc}$ and tumour angiogenesis, cell proliferation, and histological grading was observed, indicating that 4F2hc may play an essential role in tumour metastasis and progression of NENs. As our study included a short follow-up period for prognosis after definitive diagnosis, we could not confirm the results of OS. It has been previously reported that $4 \mathrm{~F} 2 \mathrm{hc}$, but not LAT1, contributes to tumour metastasis and survival at advanced stages of the disease rather than the early stages (19). Although it remains unclear why the expression of 4F2hc plays an important role in tumour progression for advanced stages, it could be explained by the strong connection between 4F2hc and lymph node metastases (19). Moreover, we previously reported that $4 \mathrm{~F} 2 \mathrm{hc}$ expression is closely correlated with glucose metabolism, hypoxia, and the mTOR 
pathway in pulmonary NETs (18). Several previous studies have identified $4 \mathrm{~F} 2 \mathrm{hc}$ as an independent prognostic factor related to poor outcomes and angiogenesis, indicating an essential role of 4F2hc expression in human neoplasms (11, $18-20,25)$. In our study, there was no statistically significant difference between $4 \mathrm{~F} 2 \mathrm{hc}$ and prognosis, but the hazard ratio of 3.15 suggests the possibility of a relationship between $4 \mathrm{~F} 2 \mathrm{hc}$ and poor prognosis. In addition, we believe that $4 \mathrm{~F} 2 \mathrm{hc}$ functions in tumour angiogenesis and malignant grading of neuroendocrine features. Further large-scale investigations are warranted to confirm the results of our study.

Our study has certain limitations. First, it utilized a retrospective approach with a limited cohort size, which may have biased the results. There was an obvious deviation among the populations of NET-G1, G2, G3, and NEC. Moreover, the primary site was predominantly the gastrointestinal tract, rather than the pancreas. Further investigation should focus on the homogenous histology and primary site to elucidate the prognostic significance of amino acid transporters. Second, we did not examine the clinicopathological significance of the expression of other amino acid transporters, such as ASCT2 or xCT, which are highly expressed in cancer cells, in addition to the expression of LAT1 and 4F2hc. A previous study demonstrated that ASCT2, xCT, LAT1, and 4F2hc, are highly expressed in tumour cells, and that ASCT2 and 4F2hc expression are closely correlated (26). These amino acid transporters may play a crucial role in tumour development and progression. Lastly, only a few patients receiving mTOR inhibitors were included. The possibility of LAT1 and 4F2he serving as predictive markers for mTOR inhibitors is interesting, and further studies are warranted to evaluate the relationship between the efficacy of mTOR inhibitors and the expression of LAT1 and 4F2hc in patients with gastrointestinal NEN.

In conclusion, this study found no association between LAT1 expression and malignancy in GEP-NENs, but demonstrated an association between 4F2hc expression and the potential for malignancy, including vascular invasion, histological grading, and Ki-67 index. Further studies are warranted to elucidate the possibility of employing $4 \mathrm{~F} 2 \mathrm{hc}$ as a biomarker to predict the outcome after treatment with mTOR inhibitors in patients with GEP-NENs.

\section{Conflicts of Interest}

Yosuke Horita has received a speaker honorarium from Ono Pharmaceutical Company, Chugai Pharmaceutical, Taiho Pharmaceutical, Eli Lilly Japan, Novartis Pharma K.K., FUJIFILM RI Pharma K.K., Bristol Myers Squibb, Takeda Pharmaceutical Company Limited, and Sanofi K.K.

Kyoichi Kaira has received research grants and a speaker honorarium from Ono Pharmaceutical Company, Boehringer Ingelheim, Chugai Pharmaceutical, Taiho Pharmaceutical, Eli Lilly Japan, and AstraZeneca.

\section{Authors' Contributions}

Conception and design: Yosuke Horita, Kyoichi Kaira, Tomonori Kawasaki; Financial support: Kyoichi Kaira, Tetsuya Hamaguchi.

\section{Acknowledgements}

The Authors would like to thank Ms. Kozue Watanabe for her great help in staining the pathological specimens. LAT1 antibody for immunohistochemistry was provided from J-Pharma Co., Ltd. Company (Yokohama, Japan). This research received no specific grant from any funding agency in the public, commercial, or notfor-profit sectors.

\section{References}

1 Kaira K, Endo M, Abe M, Nakagawa K, Ohde Y, Okumura T, Takahashi T, Murakami H, Tsuya A, Nakamura Y, Naito T, Hayashi I, Serizawa M, Koh Y, Hanaoka H, Tominaga H, Oriuchi N, Kondo H, Nakajima T and Yamamoto N: Biologic correlation of 2-[18F]-fluoro-2-deoxy-D-glucose uptake on positron emission tomography in thymic epithelial tumors. J Clin Oncol 28(23): 3746-3753, 2010. PMID: 20625125. DOI: 10.1200/JCO.2009.27.4662

2 Yanagida O, Kanai Y, Chairoungdua A, Kim DK, Segawa H, Nii T, Cha SH, Matsuo H, Fukushima J, Fukasawa Y, Tani Y, Taketani Y, Uchino H, Kim JY, Inatomi J, Okayasu I, Miyamoto K, Takeda E, Goya T and Endou H: Human L-type amino acid transporter 1 (LAT1): characterization of function and expression in tumor cell lines. Biochim Biophys Acta 1514(2): 291-302, 2001. PMID: 11557028. DOI: 10.1016/s0005-2736(01)00384-4

3 Kanai Y, Segawa H, Miyamoto Ki, Uchino H, Takeda E and Endou $\mathrm{H}$ : Expression cloning and characterization of a transporter for large neutral amino acids activated by the heavy chain of 4F2 antigen (CD98). J Biol Chem 273(37): 2362923632, 1998. PMID: 9726963. DOI: 10.1074/jbc.273.37.23629

4 Kaira K, Oriuchi N, Imai H, Shimizu K, Yanagitani N, Sunaga N, Hisada T, Tanaka S, Ishizuka T, Kanai Y, Endou H, Nakajima $\mathrm{T}$ and Mori M: Prognostic significance of L-type amino acid transporter 1 expression in resectable stage I-III nonsmall cell lung cancer. Br J Cancer 98(4): 742-748, 2008. PMID: 18253116. DOI: $10.1038 /$ sj.bjc.6604235

5 Fuchs BC and Bode BP: Amino acid transporters ASCT2 and LAT1 in cancer: partners in crime? Semin Cancer Biol 15(4): 254-266, 2005. PMID: 15916903. DOI: 10.1016/j.semcancer. 2005.04.005

6 Furuya M, Horiguchi J, Nakajima H, Kanai Y and Oyama T: Correlation of L-type amino acid transporter 1 and CD98 expression with triple negative breast cancer prognosis. Cancer Sci 103(2): 382-389, 2012. PMID: 22077314. DOI: 10.1111/ j.1349-7006.2011.02151.x

7 Nawashiro H, Otani N, Shinomiya N, Fukui S, Ooigawa H, Shima K, Matsuo H, Kanai Y and Endou H: L-type amino acid transporter 1 as a potential molecular target in human astrocytic tumors. Int J Cancer 119(3): 484-492, 2006. PMID: 16496379. DOI: $10.1002 / \mathrm{ijc} .21866$

8 Sakata T, Ferdous G, Tsuruta T, Satoh T, Baba S, Muto T, Ueno A, Kanai Y, Endou $\mathrm{H}$ and Okayasu I: L-type amino-acid transporter 1 as a novel biomarker for high-grade malignancy in 
prostate cancer. Pathol Int 59(1): 7-18, 2009. PMID: 19121087. DOI: $10.1111 / \mathrm{j} .1440-1827.2008 .02319 . x$

9 Kaira K, Sunose Y, Arakawa K, Ogawa T, Sunaga N, Shimizu $\mathrm{K}$, Tominaga $\mathrm{H}$, Oriuchi $\mathrm{N}$, Itoh $\mathrm{H}$, Nagamori $\mathrm{S}$, Kanai $\mathrm{Y}$, Segawa A, Furuya M, Mori M, Oyama T and Takeyoshi I: Prognostic significance of L-type amino-acid transporter 1 expression in surgically resected pancreatic cancer. $\mathrm{Br} \mathrm{J}$ Cancer 107(4): 632-638, 2012. PMID: 22805328. DOI: 10.1038/bjc. 2012.310

10 Imai H, Kaira K, Oriuchi N, Shimizu K, Tominaga H, Yanagitani N, Sunaga N, Ishizuka T, Nagamori S, Promchan K, Nakajima $\mathrm{T}$, Yamamoto N, Mori M and Kanai Y: Inhibition of L-type amino acid transporter 1 has antitumor activity in non-small cell lung cancer. Anticancer Res 30(12): 4819-4828, 2010. PMID: 21187458 .

11 Kaira K, Oriuchi N, Takahashi T, Nakagawa K, Ohde Y, Okumura T, Murakami H, Shukuya T, Kenmotsu H, Naito T, Kanai Y, Endo M, Kondo H, Nakajima T and Yamamoto N: LAT1 expression is closely associated with hypoxic markers and mTOR in resected non-small cell lung cancer. Am J Transl Res 3(5): 468-478, 2011. PMID: 22046488.

12 Kaira K, Oriuchi N, Imai H, Shimizu K, Yanagitani N, Sunaga N, Hisada T, Kawashima O, Iijima H, Ishizuka T, Kanai Y, Endou H, Nakajima T and Mori M: Expression of L-type amino acid transporter 1 (LAT1) in neuroendocrine tumors of the lung. Pathol Res Pract 204(8): 553-561, 2008. PMID: 18440724. DOI: 10.1016/j.prp.2008.02.003

13 Ogawa H, Kaira K, Motegi Y, Yokobori T, Takada T, Katoh R, Osone K, Takahashi R, Katayama C, Oyama T, Kanai Y, Yao T, Asao T, Kuwano $\mathrm{H}$ and Shirabe K: Role of amino acid transporter expression as a prognostic marker in patients with surgically resected colorectal cancer. Anticancer Res 39(5): 2535-2543, 2019. PMID: 31092450. DOI: 10.21873/anticanres. 13375

14 Honjo H, Kaira K, Miyazaki T, Yokobori T, Kanai Y, Nagamori $\mathrm{S}$, Oyama T, Asao $\mathrm{T}$ and Kuwano H: Clinicopathological significance of LAT1 and ASCT2 in patients with surgically resected esophageal squamous cell carcinoma. J Surg Oncol 113(4): 381-389, 2016. PMID: 26936531. DOI: 10.1002/jso.24160

15 Lloyd RV, Osamura RY, Klöppel G and Rosai J: WHO Classification of Tumors of Endocrine Organs. WHO Classification of Tumors, $4^{\text {th }}$ Edition, Volume 10. Lyon, France, IARC, 2017.

16 WHO Classification of Tumors Editorial Board: Digestive System Tumors. WHO Classification of Tumors, $5^{\text {th }}$ Edition, Volume 1. Lyon, France, IARC, 2019.

17 Travis WD, Brambilla E, Nicholson AG, Yatabe Y, Austin JHM, Beasley MB, Chirieac LR, Dacic S, Duhig E, Flieder DB, Geisinger K, Hirsch FR, Ishikawa Y, Kerr KM, Noguchi M, Pelosi G, Powell CA, Tsao MS, Wistuba I and WHO Panel: The 2015 World Health Organization classification of lung tumors: Impact of genetic, clinical and radiologic advances since the 2004 classification. J Thorac Oncol 10(9): 1243-1260, 2015. PMID: 26291008. DOI: 10.1097/JTO.0000000000000630

18 Kaira K, Ohde Y, Endo M, Nakagawa K, Okumura T, Takahashi T, Murakami H, Tsuya A, Nakamura Y, Naito T, Kondo H, Nakajima $\mathrm{T}$ and Yamamoto N: Expression of 4F2hc (CD98) in pulmonary neuroendocrine tumors. Oncol Rep 26(4): 931-937, 2011. PMID: 21750865. DOI: 10.3892/or.2011.1384
19 Kaira K, Oriuchi N, Imai H, Shimizu K, Yanagitani N, Sunaga N, Hisada T, Kawashima O, Kamide Y, Ishizuka T, Kanai Y, Nakajima T and Mori M: CD98 expression is associated with poor prognosis in resected non-small-cell lung cancer with lymph node metastases. Ann Surg Oncol 16(12): 3473-3481, 2009. PMID: 19777189. DOI: 10.1245/s10434-009-0685-0

20 Kaira K, Takahashi T, Abe M, Akamatsu H, Nakagawa K, Ohde Y, Okumura T, Murakami H, Tsuya A, Nakamura Y, Naito T, Kondo $\mathrm{H}$, Nakajima T, Endo $\mathrm{M}$ and Yamamoto $\mathrm{N}$ : CD98 expression is associated with the grade of malignancy in thymic epithelial tumors. Oncol Rep 24(4): 861-867, 2010. PMID: 20811665. DOI: $10.3892 /$ or.2010.861

21 Briest F and Grabowski P: PI3K-AKT-mTOR-signaling and beyond: the complex network in gastroenteropancreatic neuroendocrine neoplasms. Theranostics 4(4): 336-365, 2014. PMID: 24578720. DOI: 10.7150/thno.7851

22 Murugan AK: mTOR: Role in cancer, metastasis and drug resistance. Semin Cancer Biol 59: 92-111, 2019. PMID: 31408724. DOI: 10.1016/j.semcancer.2019.07.003

23 Yao JC, Shah MH, Ito T, Bohas CL, Wolin EM, Van Cutsem E, Hobday TJ, Okusaka T, Capdevila J, de Vries EG, Tomassetti P, Pavel ME, Hoosen S, Haas T, Lincy J, Lebwohl D, Öberg K and RAD001 in Advanced Neuroendocrine Tumors, Third Trial (RADIANT-3) Study Group: Everolimus for advanced pancreatic neuroendocrine tumors. N Engl J Med 364(6): 514523, 2011. PMID: 21306238. DOI: 10.1056/NEJMoa1009290

24 Yao JC, Fazio N, Singh S, Buzzoni R, Carnaghi C, Wolin E, Tomasek J, Raderer M, Lahner H, Voi M, Pacaud LB, Rouyrre N, Sachs C, Valle JW, Fave GD, Van Cutsem E, Tesselaar M, Shimada Y, Oh DY, Strosberg J, Kulke MH, Pavel ME and RAD001 in Advanced Neuroendocrine Tumours, Fourth Trial (RADIANT-4) Study Group: Everolimus for the treatment of advanced, non-functional neuroendocrine tumours of the lung or gastrointestinal tract (RADIANT-4): a randomised, placebocontrolled, phase 3 study. Lancet 387(10022): 968-977, 2016. PMID: 26703889. DOI: 10.1016/S0140-6736(15)00817-X

25 Satoh T, Kaira K, Takahashi K, Takahashi N, Kanai Y, Asao T, Horiguchi $J$ and Oyama T: Prognostic significance of the expression of CD98 (4F2hc) in gastric cancer. Anticancer Res 37(2): 631-636, 2017. PMID: 28179310. DOI: 10.21873/ anticanres. 11357

26 Toyoda M, Kaira K, Ohshima Y, Ishioka NS, Shino M, Sakakura K, Takayasu Y, Takahashi K, Tominaga H, Oriuchi N, Nagamori S, Kanai Y, Oyama T and Chikamatsu K: Prognostic significance of amino-acid transporter expression (LAT1, ASCT2, and xCT) in surgically resected tongue cancer. Br J Cancer 110(10): 25062513, 2014. PMID: 24762957. DOI: 10.1038/bjc.2014.178 